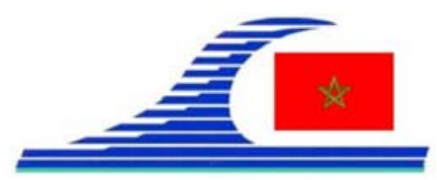

Conférence Méditerranéenne Côtière et Maritime

EDITION 2, TANGER, MAROC (2011)

Coastal and Maritime Mediterranean Conference

Disponible en ligne - http://www.paralia.fr - Available online

\title{
Identification bayésienne des paramètres de modélisation de la pénétration des ions chlorure dans le béton
}

\author{
Emilio BASTIDAS-ARTEAGA ${ }^{1}$, Manh Tuan NGUYEN ${ }^{1}$, \\ Franck SCHOEFS ${ }^{1}$, Stéphanie BONNET ${ }^{1}$
}

\author{
1. LUNAM Université, Université de Nantes-Ecole Centrale Nantes, GeM, Institut de \\ Recherche en Génie Civil et Mécanique, CNRS UMR 6183, Nantes, France. \\ emilio.bastidas@univ-nantes.fr
}

\section{Résumé :}

La présence des ions chlorure, notamment en sites maritimes, est l'une des causes majeures provoquant la dégradation des structures en béton armé par la corrosion de leurs armatures. Par conséquent, la prédiction de la pénétration des chlorures dans le béton est une composante majeure pour planifier et quantifier correctement les interventions d'inspection et maintenance sur la structure. La prédiction a besoin des paramètres matériaux et environnementaux pertinents déterminés à partir des mesures expérimentales. L'objectif principal de cet article est de mettre au point une méthode pour l'identification des paramètres de modélisation de diffusion des ions chlorure dans le béton à partir de mesures en utilisant un modèle d'analyse inverse reposant sur des principes probabilistes. Les résultats de l'identification sur un échantillon numérique indiquent que l'approche bayésienne pourrait être utile pour identifier les paramètres d'un modèle à partir des données réelles.

\section{Mots-clés :}

Pénétration de chlorures dans le béton - Corrosion - Analyse inverse - Réseaux bayésiens - Identification

\section{Introduction}

La corrosion des armatures est l'une des causes principales de la dégradation des structures en béton armé. Cette corrosion peut se produire par l'exposition aux chlorures ou au dioxyde de carbone présents dans l'environnement. Cette étude se centre sur la pénétration des ions chlorures dans le béton qui déclenche la corrosion quand une concentration seuil de chlorures atteint les armatures.

La prédiction de la pénétration des ions chlorure dans le béton par modélisation est la composante clé permettant de planifier et de quantifier correctement les interventions (inspection et maintenance) sur une structure exposée à cet environnement agressif. Etant donné que le processus de pénétration des chlorures est très complexe (BASTIDAS-ARTEAGA et al., 2011), cette modélisation doit s'appuyer sur des mesures expérimentales afin de réaliser des prédictions pertinentes. Les mesures expérimentales servent à déterminer les profils de teneur en chlorures en un point de la 
La connaissance de la Mer :

un vecteur du développement durable en Méditerranée

structure. Les résultats de ces mesures sont très variables et l'erreur de mesure est significative (BONNET et al., 2009). Par conséquent, un grand nombre de mesures est nécessaire pour évaluer des paramètres pertinents pour la modélisation. Tenant compte que ces essais sont coûteux et difficiles à mettre en œuvre une optimisation est nécessaire. Elle ne pourra reposer à l'avenir que sur une approche d'analyse de risques intégrant les incertitudes. C'est pourquoi on propose une analyse inverse dans un cadre probabiliste à partir de réseaux bayésiens afin de déterminer les paramètres du modèle prenant en compte l'aléa réel du béton et des mécanismes de migration des chlorures.

La section 2 décrit le modèle analytique de pénétration des chlorures et pose le problème dans un cadre bayésien. Afin de valider la méthode bayésienne nous avons utilisé des échantillons numériques obtenus à partir de simulations de Monte-Carlo (section 3). Enfin, la section 4 présentera un exemple de la démarche proposée pour l’identification des paramètres du modèle de pénétration des chlorures.

\section{Application du théorème de Bayes au problème de diffusion}

La deuxième loi de diffusion de Fick est généralement utilisée pour modéliser le flux chlorures dans le béton (TUUTTI, 1982). En supposant que le béton est un matériau homogène, isotrope, saturé et soumis à une concentration de chlorures à la surface constante, $C_{s}$, la concentration des ions chlorure libres à la profondeur $x$ et temps $t, C_{x t}$ devient :

$$
C_{x t}=C_{s}\left[1-\operatorname{erf}\left(\frac{x}{2 \sqrt{D t}}\right)\right]
$$

où $D$ est le coefficient de diffusion effective des ions chlorures et erf(·) est la fonction d'erreur. Le théorème de Bayes peut s'utiliser pour calculer les distributions de probabilité des variables aléatoires (NAÏM et al., 2007). En considérant que $D$ et $C_{s}$ sont les deux variables aléatoires indépendantes à identifier, la probabilité d'estimer une concentration en chlorures à un point et à un temps donnés $p\left(C_{x t}\right)$ s'écrit (NGUYEN, 2007) :

$$
p\left(C_{x t}\right)=\sum_{D, C s} p\left(C_{x t} \mid D, C_{s}\right) p\left(D, C_{s}\right) \quad \text {, avec : } p\left(D, C_{s}\right)=p(D) p\left(C_{s}\right)
$$

Dans l'équation 2, la probabilité conditionnelle $\mathrm{p}\left(C_{x t} \mid D, C_{s}\right)$ doit être déjà connue. Elle renvoie au conditionnement de la quantité de chlorure en un point aux caractéristiques matériaux : elle rend ainsi compte, sous forme purement probabiliste, des mécanismes de transferts tels que modélisés en (1). Sa détermination est l'objet du calcul de la table de probabilités conditionnelles du réseau, à partir du mécanisme choisi (ici (1)) ou de données expertes. Une fois connue $\mathrm{p}\left(C_{x t}\right)$, les lois a posteriori (lois à identifier) peuvent être calculées simplement à partir d'un ensemble de mesures de $C_{x t}$. Si $\mathrm{p}\left(C_{x t} \mid \mathrm{o}\right)$ est la loi de $C_{x t}$ sachant les observations o (mesures de $C_{x t}$ ), l'application du théorème de Bayes donne : 


$$
\begin{array}{ll}
p(D \mid o)=p\left(D \mid C_{x t}\right) p\left(C_{x t} \mid o\right) & \text {, avec : p }\left(D \mid C_{x t}\right)=\frac{p\left(C_{x t} \mid D\right) p(D)}{p\left(C_{x t}\right)} \\
p\left(C_{s} \mid o\right)=p\left(C_{s} \mid C_{x t}\right) p\left(C_{x t} \mid o\right) & \text {, avec: } p\left(C_{s} \mid C_{x t}\right)=\frac{p\left(C_{x t} \mid C_{s}\right) p\left(C_{s}\right)}{p\left(C_{x t}\right)}
\end{array}
$$

La détermination des probabilités conditionnelles s'effectue par apprentissage et inférence à l'aide du logiciel de réseaux bayésiens avec le logiciel Netica®.

\section{Obtention des échantillons synthétiques}

L'actualisation bayésienne donne des informations a posteriori qui considèrent des observations réelles du phénomène étudié appelées évidences. Ces évidences doivent s'obtenir à partir des mesures expérimentales. Toutefois, afin de tester la performance de notre réseau et d'optimiser sa configuration, nous utilisons des observations "numériques" obtenues à partir de distributions de $D$ et $C_{s}$ connues. On suppose donc que ces variables aléatoires suivent des lois normales avec les paramètres présentés dans le tableau 1. On notera que pour des applications pratiques, les distributions sont en fait des distributions s'approchant de loi log-normales. Avec ces paramètres on génère 3000 valeurs aléatoires pour $C_{s}$ et $D$ en utilisant des simulations de Monte-Carlo. Puis, pour chaque jeu de valeurs, on calcule pour deux échéances (10 et 20 ans) et trois profondeurs (1, 4 et $8 \mathrm{~cm}$ ) les concentrations $C_{x t}$ à l'aide de l'équation 1 . Ces valeurs simulent des mesures (profils de chlorures) prélevées après 10 et 20 ans d'exposition. Enfin, on calcule la probabilité que $C_{x t}$ appartienne à un intervalle donné pour profondeurs différentes. Le tableau 2 présente trois évidences numériques calculées à partir des simulations pour $t=10$ ans. Les résultats sont donnés en termes de probabilité d'appartenance à chaque intervalle.

Tableau 1. Paramètres initiaux pour la génération des observations synthétiques.

\begin{tabular}{llll}
\hline$\mu_{C S}\left(\mathbf{k g} / \mathbf{m}^{3}\right)$ & $\sigma_{C s}\left(\mathbf{k g} / \boldsymbol{m}^{3}\right)$ & $\mu_{\boldsymbol{D}}\left(\boldsymbol{m}^{2} / \mathbf{s}\right)$ & $\sigma_{D}\left(\boldsymbol{m}^{2} / \mathbf{s}\right)$ \\
\hline 5 & 1 & $3,00 \times 10^{-12}$ & $3,00 \times 10^{-13}$ \\
\hline
\end{tabular}

\begin{tabular}{|c|c|c|c|c|c|c|c|}
\hline $\begin{array}{l}\text { Intervalle } \\
\left(\mathrm{kg} / \mathrm{m}^{3}\right)\end{array}$ & $x=1 \mathrm{~cm}$ & $x=4 \mathrm{~cm}$ & $x=8 \mathrm{~cm}$ & $\begin{array}{l}\text { Intervalle } \\
\left(\mathrm{kg} / \mathrm{m}^{3}\right)\end{array}$ & $x=1 \mathrm{~cm}$ & $x=4 \mathrm{~cm}$ & $x=8 \mathrm{~cm}$ \\
\hline $0,0-0,6$ & 0 & 0,00033 & 0,992333 & $4,8-5,4$ & 0,1380 & 0 & 0 \\
\hline $0,6-1,2$ & 0,0003 & 0,05467 & 0,007667 & $5,4-6,0$ & 0,0500 & 0 & 0 \\
\hline $1,2-1,8$ & 0,0017 & 0,46333 & 0 & $6,0-6,6$ & 0,0090 & 0 & 0 \\
\hline $1,8-2,4$ & 0,0147 & 0,42667 & 0 & $6,6-7,2$ & 0,0010 & 0 & 0 \\
\hline $2,4-3,0$ & 0,0757 & 0,05433 & 0 & $7,2-7,8$ & 0 & 0 & 0 \\
\hline $3,0-3,6$ & 0,1810 & 0,00067 & 0 & $7,8-8,4$ & 0 & 0 & 0 \\
\hline $3,6-4,2$ & 0,2666 & 0 & 0 & $8,4-9,0$ & 0 & 0 & 0 \\
\hline $4,2-4,8$ & 0,2620 & 0 & 0 & Somme & 1 & 1 & 1 \\
\hline
\end{tabular}

Tableau 2. Evidence numérique à $t=10$ ans et pour trois profondeurs. 
La connaissance de la Mer :

un vecteur du développement durable en Méditerranée

\section{Identification de $D$ et $C_{s}$}

Cette partie présente la démarche utilisée dans l'identification de la moyenne et de l'écart-type de $D$ et de $C_{s}$ à partir des évidences numériques. La figure 1 montre la configuration du réseau adopté. On considère un modèle avec 6 nœuds d'enfant $C_{x t}$ et 2 nœuds parent $\left(C_{s}\right.$ et $\left.D\right)$. Les profondeurs considérées pour chaque échéance sont :

- $t=10$ ans : profondeurs $x=1 \mathrm{~cm}, 4 \mathrm{~cm}$ et $8 \mathrm{~cm}$

- $t=20$ ans : profondeurs $x=1 \mathrm{~cm}, 6 \mathrm{~cm}$ et $12 \mathrm{~cm}$

Les algorithmes des réseaux bayésiens ne gèrent, en réalité, que les variables aléatoires discrètes. Puisque les variables $D$ et $C_{s}$ sont continues, il faut d'abord les discrétiser. Pour $C_{s}$ nous avons choisi 16 états pour l'intervalle $[1 ; 9]\left(\mathrm{kg} / \mathrm{m}^{3}\right)$. Pour $D$ nous avons considéré 20 états variant entre $\left[3 \times 10^{-13} ; 6,3 \times 10^{-12}\right]\left(\mathrm{m}^{2} / \mathrm{s}\right)$.

Les tables de probabilités conditionnelles sont établies en utilisant l'équation 1 . La transformation de l'équation en tables de probabilités s'effectue par la méthode de simulation (tirage aléatoire) avec le logiciel Netica ${ }^{\circledR}$.

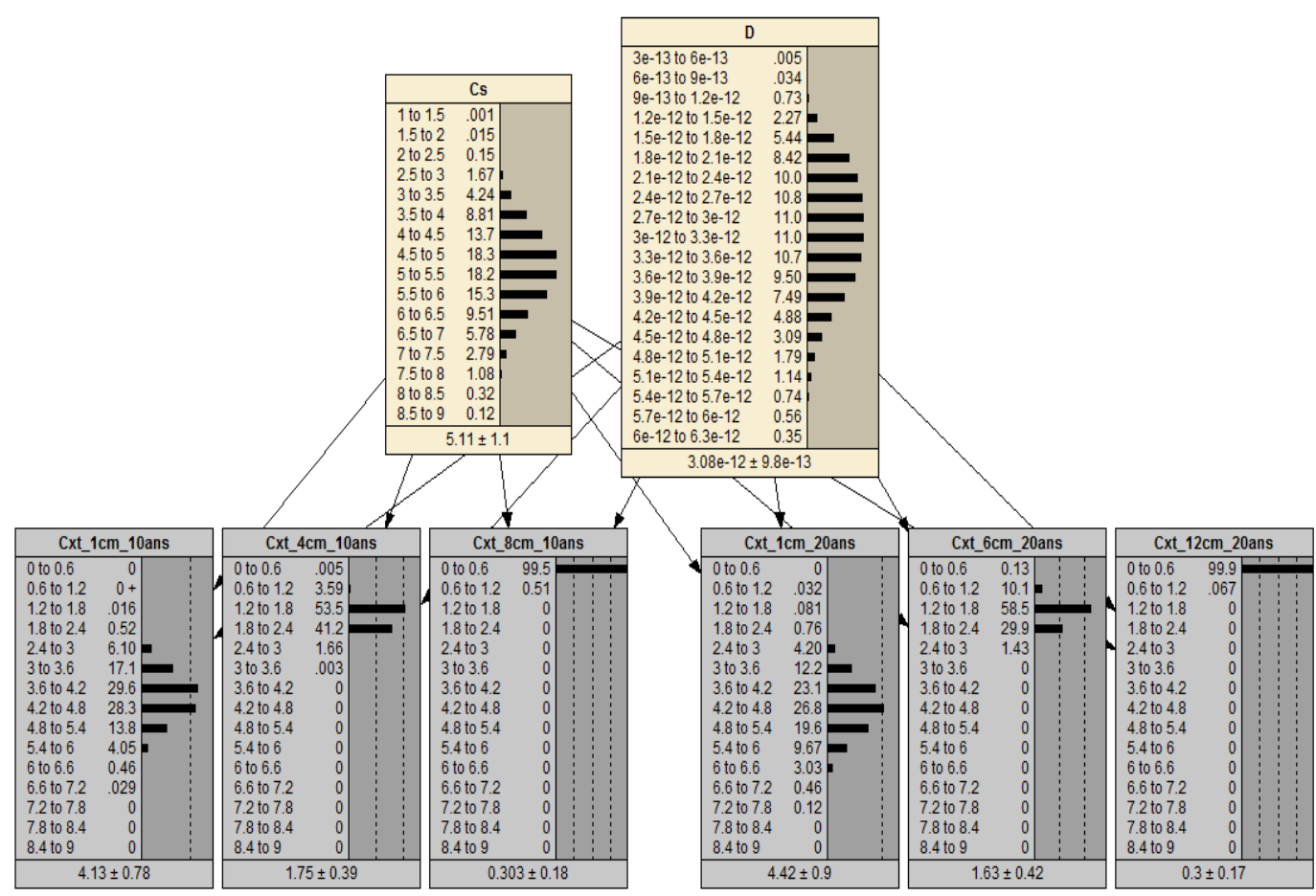

Figure 1. Réseau bayésien adopté.

Dans la première étape de l'identification on suppose que les variables $C_{s}$ et $D$ suivent une distribution uniforme (distribution a priori). Cette hypothèse est pénalisante en termes de temps de convergence (nombre d’itérations) mais évite de faire la moindre hypothèse sur la position du mode de la distribution. Lorsque les évidences numériques sont introduites dans le réseau, il est possible de calculer les distributions a posteriori de $C_{s}$ et $D$. Pour la première introduction des évidences les distributions de $C_{s}$ et $D$ 
identifiées a posteriori sont très différentes des valeurs théoriques utilisées dans la génération des variables aléatoires (tableau 1). En particulier pour l'écart-type des variables aléatoires (figure 1, itération 0). Afin d'améliorer l'estimation, nous utilisons des itérations successives où les distributions a posteriori de l'itération $i$ seront utilisées comme distribution a priori pour itération $i+1$. Les itérations sont toujours réalisées avec les mêmes évidences numériques.

La figure 2 présente l'évolution de la moyenne et de l'écart-type $C_{s}$ et $D$ après 3 itérations. Pour l'itération "0", les moyennes et les écart-types identifiés sont différents des valeurs théoriques présentées dans le tableau 1. Toutefois, dans tous les cas, la moyenne et l'écart-type s'approchent des valeurs théoriques lorsque le nombre d'itérations augmente.

La figure 3 compare les profils de teneur en ions chlorure simulés (points) avec le profil moyen et ses quantiles à 5\% et $95 \%$ identifiés après 3 itérations. On observe que la plupart des profils mesurés se trouvent dans la zone correspondant à $90 \%$ de probabilité. Par conséquent, il est possible de conclure que la configuration du réseau utilisée pour l’identification bayésienne pourrait être utile pour identifier des paramètres d'un modèle à partir des données réelles.
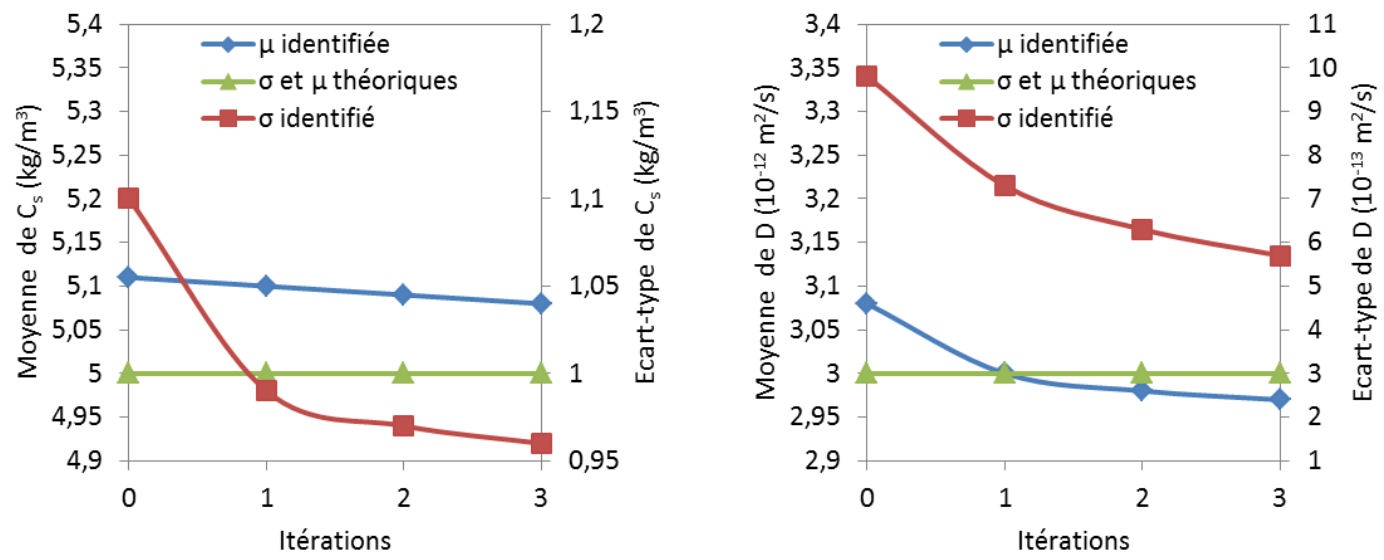

Figure 2. Evolution de la moyenne et de l'écart-type de $C_{s}$ et $D$ avec des itérations.

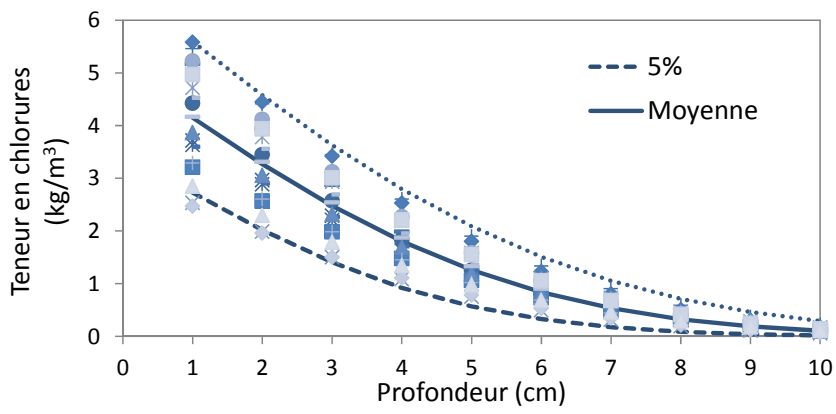

Figure 3. Comparaison entre les profils simulés (points) et la moyenne et ses quantiles identifiés. 
La connaissance de la Mer :

un vecteur du développement durable en Méditerranée

\section{Conclusions}

Les ions chlorure ont clairement été identifiés comme l'élément déclencheur du processus chimique menant à la corrosion des armatures du béton armé en site maritime. Par conséquent, la prédiction de la pénétration des ions chlorure dans le béton est nécessaire pour une gestion optimale des ouvrages. Cette prédiction doit s'appuyer sur des mesures expérimentales qui sont généralement coûteuses. Nous allons donc cherché une méthode pour valoriser ces mesures à travers l'identification bayésienne des paramètres d'un modèle de diffusion. L'approche bayésienne peut actualiser des informations du modèle par une méthode itérative. C’est-à-dire, la méthode bayésienne peut profiter efficacement du nombre limité de mesures expérimentales. Les résultats de l'identification sur des mesures numériques (simulations de Monte-Carlo) indiquent que les valeurs identifiées sont proches des valeurs théoriques. La perspective de cette étude s'inscrit principalement dans l'identification des paramètres à partir des mesures réelles.

\section{Remerciements :}

Les auteurs remercient l'état français et la région Pays de la Loire pour leur soutien financier du projet MAREO et la communauté européenne pour le financement du projet Duratinet (www.duratinet.org).

\section{Références}

BASTIDAS-ARTEAGA E., CHATEAUNEUF A., SÁNCHEZ-SILVA M., BRESSOLETTE P., SCHOEFS, F. (2011). A comprehensive probabilistic model of chloride ingress in unsaturated concrete. Engineering Structures, Vol. 33, pp. 720-730. doi:10.1016/j.engstruct.2010.11.008

BONNET S., SCHOEFS F., RICARDO J., SALTA M. (2009). Effect of error measurements of chloride profiles on reliability assessment. 10th International Conference on Structural Safety and Reliability, Japon.

NAÏM P., WUILLEMIN P.H, LERAY P., POURRET O., BECKER A. (2007). Les réseaux bayésiens, Eyrolles 3ème édition.

NGUYEN X.S. (2007). Algorithmes probabilistes appliqués à la durabilité et à la mécanique des ouvrages de génie civil. Thèse de doctorat, INSA de Toulouse, France.

TUUTTI K. (1982). Corrosion of steel in concrete. CBI Report F 482, Swedish Cement and Concrete Institute, Stockholm. 